\section{Prostaglandins and their intermediates}

from a Correspondent

An international conference on prostaglandins was held in Florence on May 26-30.

Arguably the most exciting findings of the whole meeting were revealed by S. Samuelsson (Karolinska Institute, Stockholm) in an introductory address. Recently Samuelsson's group found (Hamberg and Samuelsson, Biochem. Biophys. Res. Commun., 61, 942; 1974 and Proc. natn. Acad. Sci. U.S.A., 71, 3400 ; 1974) that $\mathrm{PGG}_{2}$ and $\mathrm{PGH}_{2}$, the endoperoxide precursors of prostaglandins (see Nature, 253, 88; 1975), can, in lungs and platelets, also be transformed into other (non-prostaglandin) products including a hemiacetal derivative 'PHD'. Now Samuelsson's group have identified a very labile intermediate in the conversion of $\mathrm{PGG}_{2}$ to PHD. This new intermediate does not have a classical prostaglandin structure and has been named 'Thromboxane' (see figure), because it is a very potent platelet aggregating agent. Since it is the first member of a new series of compounds and contains two double bonds it was further designated 'Thromboxane $\mathrm{A}_{2}$ ': its metabolite PHD now becomes 'Thromboxane $\mathbf{B}_{2}$ '.

Thromboxane $\mathrm{A}_{2}$ has at least two other interesting properties; its half-life in aqueous solutions is about $30 \mathrm{~s}$, and it strongly contracts strips of rabbit aorta. As a product of the prostaglandin biosynthesis cascade its generation is of course blocked by aspirin. These properties make it very likely that Thromboxane $A_{2}$ is in fact 'RCS'-the mysterious rabbit aorta contracting substance (released from lungs during anaphylaxis), first described by Piper and Vane in 1969 (Nature, 233, 29). The importance of the endoperoxide system in platelet aggregation was emphasised by the Karolinska group's discovery of a subject deficient in the cyclo-oxygenase system (which converts arachidonic acid to $\mathrm{PGG}_{2}$ ). Platelets from this subject showed reduced sensitivity to the aggregating action of adrenaline and collagen, but were aggregated by $\mathrm{PGG}_{2}$. The idea that the endoperoxides themselves, or their nonprostaglandin end products, may be important in other systems was later reinforced by $M$. Hamberg et al. (Karolinska Institute) who found elevated levels of endoperoxide and Thromboxane metabolites in perfúsed guinea-pig lung during anaphylaxis; $\mathrm{PGG}_{2}$ and $\mathrm{H}_{2}$ also had much more marked bronchoconstrictor activity than $\mathrm{PGF}_{2} \alpha$. Both pieces of evidence suggested that the endoperoxides (or their non-prostaglandin metabolites) could be important in the anaphylactic reaction. J. Vane (Wellcome Laboratories) presented evidence that $\mathrm{PGG}_{2}$ and $\mathrm{PGH}_{2}$ potentiated the inflammation produced in the rat paw by stimuli such as Carraggeenin.

The question of the cellular origin of the substrate required for prostaglandin biosynthesis received some attention, though not perhaps as much as it deserved. Phosphatidylinositol was definitely implicated as a source of arachidonate for prostaglandin biosynthesis in the pig thyroid undergoing TSH stimulation (B. Haye, S. Champion and C. Jaquemin; Reims-Cédex, France) and in platelets exposed to a variety of aggregating agents (Iacona and Schoene, US Dept. of Agriculture, Beltsville). Some data from Vane's group suggested that phosphatidylcholine was the source of the arachidonic acid liberated during trauma of spleen tissue, and demonstrated that inhibition of phospholipase $\mathrm{A}_{2}$ could prevent this initial step in the generation of prostaglandins from occurring.

The section dealing with metabolism of prostaglandins aroused less interest: prostaglandin 15-hydroxy-dehydrogenase (PGDH) has been successfully extracted and purified from a variety of sources allowing good kinetic data to be obtained. One particularly ingenious and successful method of purifying the enzyme-by affinity chromatography using prostaglandins

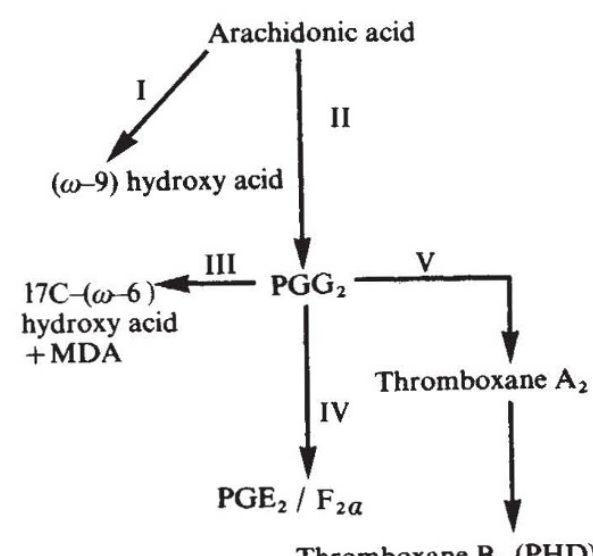

Alternative pathways of arachidonic acid metabolism in platelets. I, Conversion by a lipoxygenase of arachidonic acid to an $\omega-9$ hydroperoxide which is subsequently reduced to the corresponding hydroxy acid. II, Conversion of arachidonic acid by a cyclo-oxygenase to $\mathbf{P G G}_{2}$. III, Conversion of $\mathrm{PGG}_{2}$ to a 17C. $(\omega-6)$ hydroxy acid and malondialdehyde. IV, Conversion of $\mathrm{PGG}_{2}$ to $\mathrm{PG} \mathrm{E}_{2} / \mathrm{F}_{2}$ ". This pathway is of minor importance in platelets. V, Conversion of $\mathrm{PGG}_{2}$ to Thromboxane $\mathrm{A}_{2}$ and Thromboxane $B_{2}$ (PHD). PHD is a hemiacetal derivative of $8(1$-hydroxy-3oxopropyl) $-9, \quad 12 \mathrm{~L}$-dihydroxy-5, 10 heptadecadienoic acid. as the ligand-was presented by $\mathrm{E}$. Oliw, I. Lunden and E. Änggard (Karolinska Institute). Throughout the conference, however, many workers were disappointed that so little attention was paid to the question of inhibiting this important enzyme: a really effective inhibitory drug would complement the use of the aspirin-like agents (inhibitors of prostaglandin biosynthesis) as a pharmacological tool to investigate the function of prostaglandins in the body. R. Flower et al. (Wellcome Laboratories) presented evidence that PGDH was a short-lived enzyme within the cell, the tissue levels falling very quickly after the administration of pnotein synthesis inhibitors.

Details of two important radioimmunoassay systems were presented at the meeting: F. Dray, B. Charbonnel and J. Maclouf (Pasteur Institute, Paris) have devised techniques which eliminate most of the artefacts associated with the measurement of $\mathrm{E}$ and F-type prostaglandins in plasma and have thus reduced the falsely high readings obtained by many early workers. Their plasma levels for E-type prostaglandins of $<5 \mathrm{pg} \mathrm{ml}^{-1}$ and for the F-type of $\leqslant 12 \mathrm{pg} \mathrm{mi}^{-1}$ are probably the lowest recorded. Because prostaglandins are metabolised so rapidly, it is often more desirable to measure the major plasma $(13,14$ dihydro-15 keto) or uninary (tetranor dioic acid) metabolites of prostaglandins. E. Granström (Karolinska Institute) presented some beautiful data on immunoassay systems designed for these purposes. The levels of metabolite were found to fluctuate throughout the oestrous cycle and were depressed during treatment with aspirin-like drugs. Confidence in the validity of both these assay systems was increased by the demonstration that the blood levels found corresponded well with those predicted from daily prostaglandin production rates.

Two clinical syndromes associated with the prostaglandin system were described: the platelet cyclo-oxygenase deficiency reported by Samuelsson's group (see above), and 'hyperprostaglandinaemia'-a syndrome probably due to a deficiency of PGDH-reported by A. Labrum, M. Lipkin and F. Dray (Univensity of Rochester, New York and the Pasteur Institute, Paris). This condition was associated with periodic headache, vomiting, abdominal pain, diarrhoea and pyrexia.

Because of the anrangement of the conference, it was not possible to attend every session, so only centain aspects of the meeting are reported here. But it was clear that the impression felt by many at the end of this exciting conference was that the physiological role of prostaglandins - and now of their intermediates too-was as elusive as ever. 